Check for updates

Cite this: RSC Adv., 2017, 7, 49290

\title{
Preparation of a flame retardant phosphorus- containing polyacrylate/ $\alpha$-zirconium phosphate nanocomposite through in situ emulsion polymerization
}

\author{
Kunquan Li, ${ }^{\text {ab }}$ Huimin Lei, ${ }^{a}$ Xingrong Zeng, (D) *a Hongqiang Li, ${ }^{a}$ Xuejun Lai ${ }^{a}$ \\ and Shengyong Chai ${ }^{\mathrm{b}}$
}

An acrylamide modified $\alpha$-zirconium phosphate (AM-ZrP) was prepared by intercalating acrylamide into the $\alpha$-zirconium phosphate ( $\alpha$-ZrP), and was utilized to synthesize a flame retardant phosphorus-containing polyacrylate/ $\alpha$-zirconium phosphate (PPA/AM-ZrP) nanocomposite via in situ emulsion polymerization. The AM-ZrP was characterized by scanning electron microscopy (SEM), X-ray diffraction (XRD), Fourier transform infrared spectroscopy (FTIR) and thermogravimetric analysis (TGA). The layered structure and morphology of AM-ZrP in the PPA matrix were studied by XRD and transmission electron microscopy (TEM). Effects of AM-ZrP content on the monomer conversion, polymerization stability, thermal properties and flame retardancy of the PPA/AM-ZrP nanocomposite were studied. The flame retardant mechanism of AM-ZrP was also discussed. Results showed that the acrylamide was successfully intercalated into the $\alpha$-zirconium phosphate. The incorporation of AM-ZrP would increase the $T_{g}$ of the composite. When 0.5 wt\% of AM-ZrP was added, the exfoliated AM-ZrP nanoplatelets were covered by a layer of PPA polymer, and well dispersed in the PPA matrix. Meanwhile, the PPA/AM-ZrP composite had a lower peak heat release rate (pHRR) and higher limiting oxygen index (LOI) than the PPA, indicating that the PPA/AM-ZrP composite possessed better flame retardancy. With the further increase of the AMZrP content to $3.0 \mathrm{wt} \%$, the AM-ZrP nanoplatelets came together, forming big aggregates and showed poor dispersion in the composite, which had adverse effects on the flame retardancy of PPA/AM-ZrP. SEM and energy dispersive $X$-ray spectroscopy (EDS) analysis suggested that the existence of AM-ZrP in the PPA/AM-ZrP composite could catalyze the carbonization process and promoted the formation of a compact char layer, which was favorable for the improvement of flame retardancy of the composite.

Received 27th June 2017

Accepted 21st September 2017

DOI: $10.1039 / \mathrm{c} 7 \mathrm{ra0} 0131 \mathrm{k}$

rsc.li/rsc-advances

\section{Introduction}

Polyacrylate emulsions have gained much interest because of their excellent weather resistance, film-forming properties, flexibility and environmental friendliness, ${ }^{\mathbf{1}, 2}$ and have been widely used in various areas, such as in coatings, adhesives and painting inks. ${ }^{3-6}$ However, polyacrylate will be easily burn when it comes into contact with a fire, which may threaten people's life and property. ${ }^{7}$ Numerous studies have been reported to develop flame retardant polyacrylate by adding flame retardant additives of phosphate compounds into the polyacrylate emulsion. Due to the incompatibility between phosphates and polymers, the physical blending of the flame retardant will

${ }^{a}$ College of Materials Science and Engineering, South China University of Technology, No 381, Wushan Road, Tianhe District, Guangzhou 510640, People's Republic of China. E-mail: psxrzeng@gmail.com; Fax: +86-20-87114248; Tel: +86-20-87114248 ${ }^{b}$ National-certified Enterprise Technology Center, Kingfa Science and Technology Co., Ltd., No.33 Kefeng Road, Science Town, Guangzhou 510663, People's Republic of China cause phase separation, which deteriorates the mechanical properties of the composite. ${ }^{\mathbf{8 9}}$ Hence, it is wise to synthesize the flame retardant polyacrylate by in situ emulsion polymerization of acrylate and phosphorus-containing monomers. In our previous work, ${ }^{\mathbf{1 0}}$ a stable phosphorus-containing polyacrylate (PPA) emulsion was successfully synthesized using acrylate and phosphorus-containing alkoxy phosphate methacrylate (PAM100) as monomers. Although the PPA latex film had better flame retardancy than the polyacrylate (PA), it could not meet the practical applications because of the low phosphorus content in the latex film. Meanwhile, the mechanical properties of the PPA latex film needed to be improved. ${ }^{11}$

Recently, the flame retardant polymer nanocomposites containing layered nanomaterials, ${ }^{12,13}$ such as layered silicates ${ }^{14}$ graphite oxide, ${ }^{15}$ layered double hydroxides ${ }^{16}$ and layered metal phosphates, ${ }^{17}$ have been widely studied. It is found that the layered nanomaterials act as a physical barrier to inhibit the transmission of heat and oxygen, and promote the free-radical chain-terminating reaction and carbon layer 
formation during combustion, resulting in the improvement of flame retardancy. The mechanical properties of the composite could be also enhanced when incorporated with layered nanomaterials. ${ }^{18-20}$

$\alpha$-Zirconium phosphate $(\alpha$-ZrP), with a layered structure consisted of zirconium atoms and phosphate group, has drawn increasing consideration on the development of flame retardant polymer nanocomposites. ${ }^{21,22}$ As a montmorillonite-like layered inorganics, the $\alpha$-ZrP nanoplatelets can act as a physical barrier to prevent the decomposed gases from escaping. ${ }^{23,24}$ Alongi found that the $\alpha$-ZrP in polyamide 6 (PA6) could reduce the total heat release (THR) and the heat release rate (HRR) during the combustion, and had synergistic effects with phosphorous based flame retardants. ${ }^{25}$ Liu synthesized an organo-modified $\alpha$ zirconium phosphate $(\mathrm{OZrP})$ and studied its effects on the flame retardancy of intumescent flame retardant (IFR) poly(lactic acid) systems. It showed that the incorporation of OZrP into composites resulted in the significant reduction of the HRR and THR, which could improve the flame retardancy of nanocomposites. ${ }^{26}$ Although the $\alpha$-ZrP was conducive to the preparation of flame retardant composites, the dispersion of nanoplatelets is a crucial factor in affecting the flammability of the nanocomposites. ${ }^{27}$ As a type of inorganic phosphates, the $\alpha$-ZrP is hard to be uniformly dispersed in polymer, and the poor dispersion of $\alpha$-ZrP in polymeric matrix may lead to the deteriorated flame retardancy and mechanical properties. ${ }^{28}$ However, the $\alpha$-ZrP can be well dispersed in water, which provides favorable condition for the preparation of polyacrylate/ $\alpha$-ZrP nanocomposite emulsion.

In this work, a modified $\alpha$-zirconium phosphate (AM-ZrP) was prepared by intercalating acrylamide into $\alpha$-zirconium phosphate $(\alpha-\mathrm{ZrP})$, and was utilized to synthesize the phosphorus-containing polyacrylate/ $\alpha$-zirconium phosphate (PPA/AM-ZrP) nanocomposite via in situ emulsion polymerization. The obtained AM-ZrP was characterized by scanning electron microscopy (SEM), X-ray diffraction (XRD), Fourier transform infrared spectroscopy (FTIR) and thermogravimetric analysis (TGA). The layered structure and morphology of AMZrP in PPA matrix were studied by XRD and transmission electron microscopy (TEM). Effects of AM-ZrP content on the monomer conversion, polymerization stability, thermal properties and flame retardancy of PPA/AM-ZrP nanocomposite were studied. The flame retardant mechanism of AM-ZrP was also discussed.

\section{Experimental}

\subsection{Materials}

Zirconyl chloride $\left(\mathrm{ZrOCl}_{2} \cdot 8 \mathrm{H}_{2} \mathrm{O}\right)$, butyl acrylate (BA, A.R.), methyl methacrylate (MMA, A.R.), acrylic acid (AA, A.R.), potassium persulfate (KPS, A.R.), methylamine aqueous solution (40 wt\%, A.R.), and sodium bicarbonate $\left(\mathrm{NaHCO}_{3}\right.$, A.R.) were purchased from Tianjin Chemical Reagent Factory (China). Acrylamide (AM, C.P.) was supplied by Shanghai Runjie Chemical Reagent Co., Ltd. (China). Phosphoric acid $\left(\mathrm{H}_{3} \mathrm{PO}_{4}\right.$, A.R.) was provided by Jiangsu Qiangsheng Chemical Co., Ltd. (China). Hydrochloric acid (HCl, A.R.) was obtained from
Guangzhou Chemical Reagent Factory (China). 2-Hydroxyethyl methacrylate phosphate (PM-1500, C.P.) was acquired from Guangzhou Jingde Chemical Reagent Co., Ltd. (China). Allyloxy nonylphenol ethoxylates ammonium sulfate (DNS-86, Tech.) was gained from Guangzhou Shuangjian Trading Co., Ltd. (China). All of the materials were used as received. The chemical structure formula of PM-1500 was shown in Fig. 1.

\subsection{Preparation and modification of $\alpha$-ZrP}

$\alpha$-ZrP was prepared by refluxing $\mathrm{ZrOCl}_{2} \cdot 8 \mathrm{H}_{2} \mathrm{O}$ in $12 \mathrm{~mol} \mathrm{~L}^{-1}$ $\mathrm{H}_{3} \mathrm{PO}_{4}$ aqueous solution at $100{ }^{\circ} \mathrm{C}$ for $24 \mathrm{~h}$ as described by Sun. ${ }^{29}$

The modification of $\alpha$-ZrP was described as follows. Firstly, $1.0 \mathrm{~g} \alpha$-ZrP was pre-dispersed in $100 \mathrm{~mL}$ distilled water under ultrasound condition for 90 minutes to achieve a uniform dispersion. Secondly, $0.18 \mathrm{~g}$ methylamine aqueous solution (40 wt\%) was dripped into the dispersion to form a M-ZrP colloidal dispersion. Subsequently, the AM aqueous solution (1.0 g AM dissolved in $100 \mathrm{~mL}$ distilled water) was added in the M-ZrP colloidal dispersion under ultrasound condition within 90 minutes, and the dispersion was stirred for another $24 \mathrm{~h}$. Finally, the product was washed with distilled water, and collected by centrifugation of three times. The AM-ZrP was obtained after drying at $75{ }^{\circ} \mathrm{C}$ for $24 \mathrm{~h}$, and ground into fine powders.

\subsection{Synthesis of PPA/AM-ZrP nanocomposite emulsion}

PPA/AM-ZrP nanocomposite emulsion was synthesized via in situ emulsion polymerization. A given mass of AM-ZrP (for the synthesis of PPA, the AM-ZrP content was $0 \mathrm{~g}$ ) was mixed with $40.0 \mathrm{~g}$ MMA, $40.0 \mathrm{~g}$ BA and $1.32 \mathrm{~g} \mathrm{AA}$, and the mixture was sonicated for $2 \mathrm{~h}$. Then, the mixed monomers were added into the aqueous solution (1.42 $\mathrm{g}$ DNS-86 and $7.12 \mathrm{~g}$ PM-1500 dissolved in $60.0 \mathrm{~g}$ distilled water) with the help of a high speed mechanical dispersion homogenizer (FJ-200, Shanghai Suoying Instruments Co., Ltd.) to form a pre-emulsion. After that, $0.72 \mathrm{~g}$ $\mathrm{NaHCO}_{3}$ and $60.0 \mathrm{~g}$ distilled water were transferred to a $250 \mathrm{~mL}$ four-necked glass flask equipped with Teflon mechanical stirrer, reflux condenser and dropping funnels, and the temperature of the reactor was raised to $80{ }^{\circ} \mathrm{C}$ in a water bath. Then the pre-emulsion was added dropwise simultaneously with KPS aqueous solution ( $0.36 \mathrm{~g}$ KPS dissolved in $20 \mathrm{~g} \mathrm{H}_{2} \mathrm{O}$ ) into the glass flask in 2.5-3 h under mechanical stirring, and the polymerization was allowed to react for another $1 \mathrm{~h}$ at $85^{\circ} \mathrm{C}$. Finally, the PPA/AM-ZrP composite emulsion was obtained after cooling down to the room temperature. The synthesized PPA/ AM-ZrP composite emulsions with different AM-ZrP content of $0.5 \mathrm{wt} \%, 1.0 \mathrm{wt} \%, 2.0 \mathrm{wt} \%$ and $3.0 \mathrm{wt} \%$ were marked as PPA/ AM-ZrP-1, PPA/AM-ZrP-2, PPA/AM-ZrP-3 and PPA/AM-ZrP-4,<smiles>C=C(C)C(=O)OCC[P+](=O)(O)O</smiles>

Fig. 1 Chemical structure formula of PM-1500. 
respectively. The procedures for the preparation of PPA/AM-ZrP nanocomposite emulsion were represented in Fig. 2.

\subsection{Characterization}

2.4.1 X-ray diffraction (XRD). The composite emulsion was added onto the silicon wafer and the sample was obtained after drying at room temperature. The X-ray diffraction pattern of the sample was recorded on X'Pert PRO (Panalytical., Netherlands) at an accelerating voltage of $40 \mathrm{kV}$ with a scanning speed of $4^{\circ}$ in the range of $2 \theta$ from $1^{\circ}$ to $50^{\circ}$ using $\mathrm{CuK} \alpha$ ray.

2.4.2 Fourier transform infrared spectroscopy (FTIR). Fourier transform infrared spectroscopy (FTIR) was operated by a Bruker Tensor 27 spectrometer (Bruker Optics, Germany) in a range from 4000 to $400 \mathrm{~cm}^{-1}$ with a spectral resolution of $4 \mathrm{~cm}^{-1}$ using $\mathrm{KBr}$ pellet method.

2.4.3 Transmission electron microscopy (TEM). The morphologies of $\alpha-Z r P$ and PPA/AM-ZrP nanocomposite were observed by JEM-2100F microscopy (JOEL Company, Japan). A microtome was utilized to prepare thin sections with $70-100 \mathrm{~nm}$ in thickness for TEM imaging. Samples were prepared based on the latex with a concentration of $0.1 \mathrm{wt} \%$ (diluted with water), dried at room temperature until the water was volatilized before testing.

2.4.4 Dynamic light scattering (DLS) test. The statistics analysis of mean dimension of $\alpha$-ZrP nanoplatelets was determined using dynamic light scattering (DLS) test, which was performed by a dynamic light scattering detector (90 plus, Brookhaven Instruments Corporation, America). Sample was diluted with water until the concentration was about $0.1 \mathrm{wt} \%$ before testing.

2.4.5 Thermogravimetric analysis (TGA). Thermogravimetric analysis was performed on a TG209 thermogravimetry (Netzsch Instruments Co., Germany) from $30{ }^{\circ} \mathrm{C}$ to $800{ }^{\circ} \mathrm{C}$ with a heating rate of $20{ }^{\circ} \mathrm{C} \mathrm{min}^{-1}$ under air atmosphere.

2.4.6 Differential scanning calorimetry (DSC). The glass transition temperature $\left(T_{\mathrm{g}}\right)$ was measured by a DSC 204-F1 differential scanning calorimetry (Netzsch Instruments Co.,
Germany) from $-50{ }^{\circ} \mathrm{C}$ to $150{ }^{\circ} \mathrm{C}$ at a heating rate of $20{ }^{\circ} \mathrm{C} \min ^{-1}$.

2.4.7 Flame retardancy tests. The limiting oxygen index test (LOI) was carried out on an HC-2 oxygen index meter (Jiangning Analysis Instrument Co., China) according to GB/T2406-1993. The samples were dried and molded in a Teflon mold at $105^{\circ} \mathrm{C}$ for $2 \mathrm{~h}$.

The cone calorimetric test (CCT) was performed on a cone calorimeter (Fire Testing Technology Co., UK) according to the ISO5660. The samples were dried and molded in a Teflon mold at $105{ }^{\circ} \mathrm{C}$ for $2 \mathrm{~h}$, and wrapped in aluminum foil and exposed horizontally to an external heat flux of $35 \mathrm{~kW} \mathrm{~m}^{-2}$.

2.4.8 Scanning electron microscopy-energy dispersive X-ray spectroscopy (SEM-EDS). SEM images of surface morphology were taken by an EVO18 scanning electron microscopy (Carl Zeiss Jena, Co., Germany). Samples were coated with a thin layer of gold before testing to prevent electric discharge. The element analysis was operated with an OXFORD INCA250 energy dispersive spectroscopy (Oxford Instruments Co., Britain) united from SEM.

2.4.9 Monomer conversion and coagulation rate. About $1 \mathrm{~g}$ latex was dried at $120{ }^{\circ} \mathrm{C}$ to constant weight, and monomer conversion was measured according to the following eqn (1),

$$
\text { Monomer conversion }=\frac{W_{1}-W_{2}}{W_{3}} \times 100 \%
$$

where $W_{1}, W_{2}$ and $W_{3}$ represented the solid weight of the latex taken after drying, the non-involatile compositions, and the monomers used for preparing the latex, respectively.

The final composite emulsion was filtered and the aggregation was collected. Coagulation rate was defined as the weight percent of the dried aggregation to the total of monomers and AM-ZrP.

\section{Results and discussion}

\subsection{Characterization of $\alpha-Z r P$ and AM-ZrP}

The SEM image and particle size distribution of $\alpha$-ZrP were shown in Fig. 3. From Fig. 3(A), the synthesized $\alpha$-ZrP particles

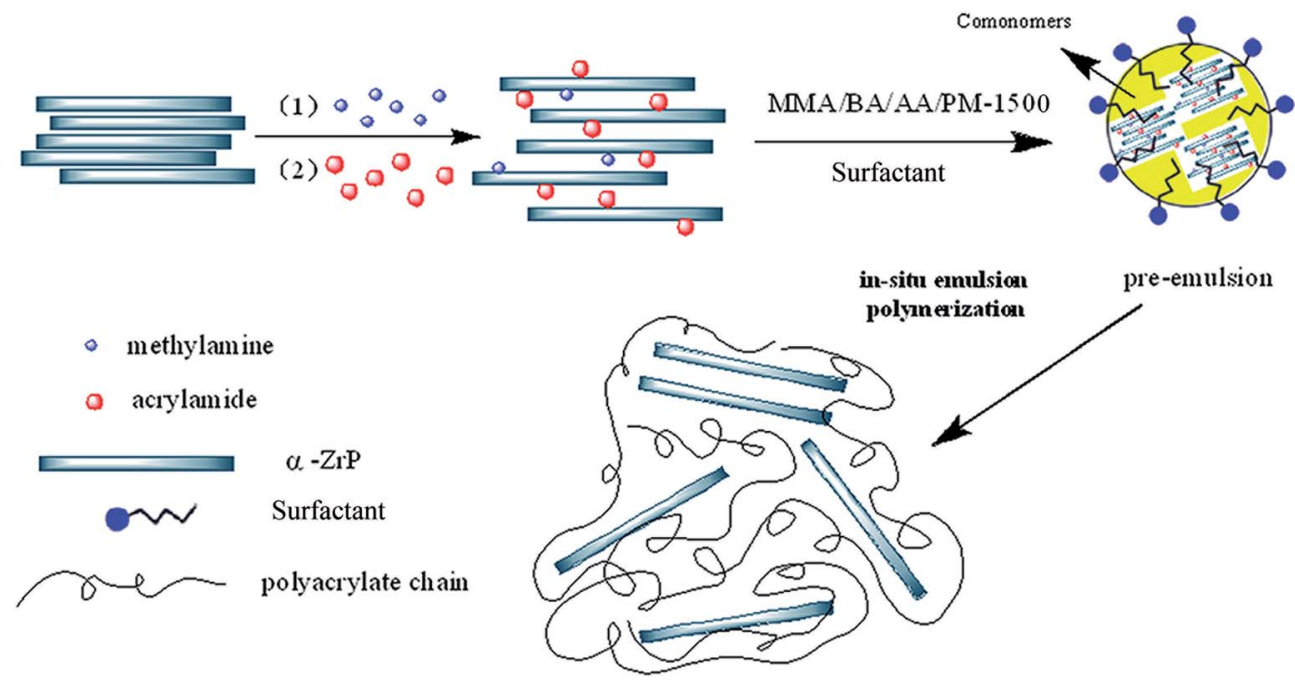

Fig. 2 Schematic illustration for the preparation of PPA/AM-ZrP nanocomposite. 

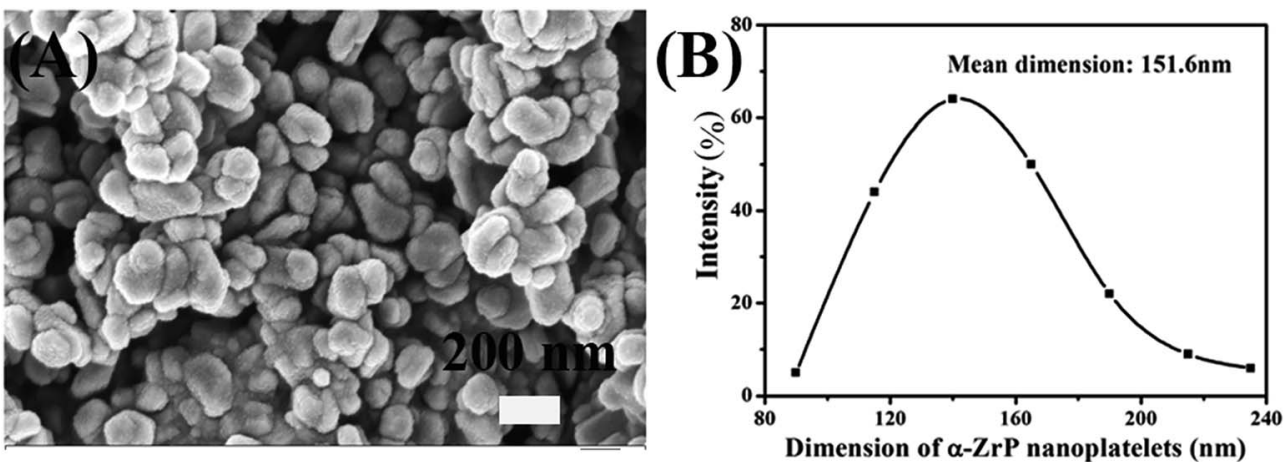

Fig. 3 SEM image and particle size distribution of $\alpha$-ZrP

irregularly distributed with a flake-like structure, and the dimension of the $\alpha$-ZrP was about 100-200 nm. From the study of Sun, ${ }^{29}$ when the concentration of phosphoric acid was $12.0 \mathrm{~mol} \mathrm{~L}^{-1}$, the obtained $\alpha$-ZrP showed platelet shape with a high aspect ratio. In Fig. 3(B), the mean dimension of $\alpha$-ZrP particles was $151.6 \mathrm{~nm}$ based on the statistics analysis between $90 \mathrm{~nm}$ and $235 \mathrm{~nm}$.

Fig. 4(A) shows the XRD spectra of $\alpha$-ZrP, methylamine modified ZrP (AM-ZrP) and AM modified ZrP (AM-ZrP). As shown in XRD spectra, the $\alpha$-ZrP displayed a 002 diffraction peak at $2 \theta=11.7^{\circ}$ with a basal interlayer spacing of $0.76 \mathrm{~nm}$. After intercalation with methylamine, the van der Waals force between the layers was weakened and the interlayer spacing of the M-ZrP increased to $0.88 \mathrm{~nm}$, which created possibility and facility for the intercalation of AM into the gallery. In the XRD spectrum of AM-ZrP, the interlayer spacing increased to $0.96 \mathrm{~nm}$, indicating that the AM was successfully intercalated inside the phosphate gallery. ${ }^{30}$ From the FTIR spectrum of $\alpha$-ZrP shown in Fig. 4(B), the peaks at $3000-3200 \mathrm{~cm}^{-1}$ and $1251 \mathrm{~cm}^{-1}$ were ascribed to the characteristic absorptions of $-\mathrm{OH}$ and $\mathrm{P}-\mathrm{OH}$ stretching vibration, respectively. While in the FTIR spectrum of AM-ZrP, the absorption band of $\mathrm{P}-\mathrm{OH}$ at $1251 \mathrm{~cm}^{-1}$ disappeared and a new absorption band at $1450 \mathrm{~cm}^{-1}$ of $\mathrm{C}-\mathrm{H}$ appeared, which suggested that the amino groups of AM had reacted with the $\mathrm{P}-\mathrm{OH}$ groups from $\alpha$-ZrP. ${ }^{31}$ Meanwhile, the existence of N-H group made the absorption bands near 3000$3200 \mathrm{~cm}^{-1}$ become wide and strong.

As shown in Fig. 5(A) and (B), the mass loss of the $\alpha$-ZrP near $143{ }^{\circ} \mathrm{C}$ was mainly caused by the loss of crystallization water between the nanoplatelets of $\alpha-\mathrm{ZrP}$, and the mass loss at $562{ }^{\circ} \mathrm{C}$ was ascribed to the dehydration condensation reaction of $\mathrm{P}-\mathrm{OH}$ inside the nanoplatelets. However, for the AM-ZrP, the mass loss at $278^{\circ} \mathrm{C}$ might be due to the deamination of amide, ${ }^{32}$ and the mass loss near $423{ }^{\circ} \mathrm{C}$ was attributed to the decomposition of AM. Meanwhile, the residue of the $\alpha-\mathrm{ZrP}$ at $800{ }^{\circ} \mathrm{C}$ was $88.3 \mathrm{wt} \%$, and it decreased to $66.8 \mathrm{wt} \%$ after intercalating with AM.

\subsection{Effects of AM-ZrP on the monomer conversion and coagulation rate}

The effects of AM-ZrP content on the monomer conversion and coagulation rate were presented in Fig. 6 . It showed that the PPA emulsion possessed good polymerization stability with a monomer conversion of $90.0 \mathrm{wt} \%$ and a low coagulation rate of $0.20 \mathrm{wt} \%$. When $0.5 \mathrm{wt} \%$ of AM-ZrP was added, the monomer conversion was raised to $92.9 \mathrm{wt} \%$ and the coagulation rate had
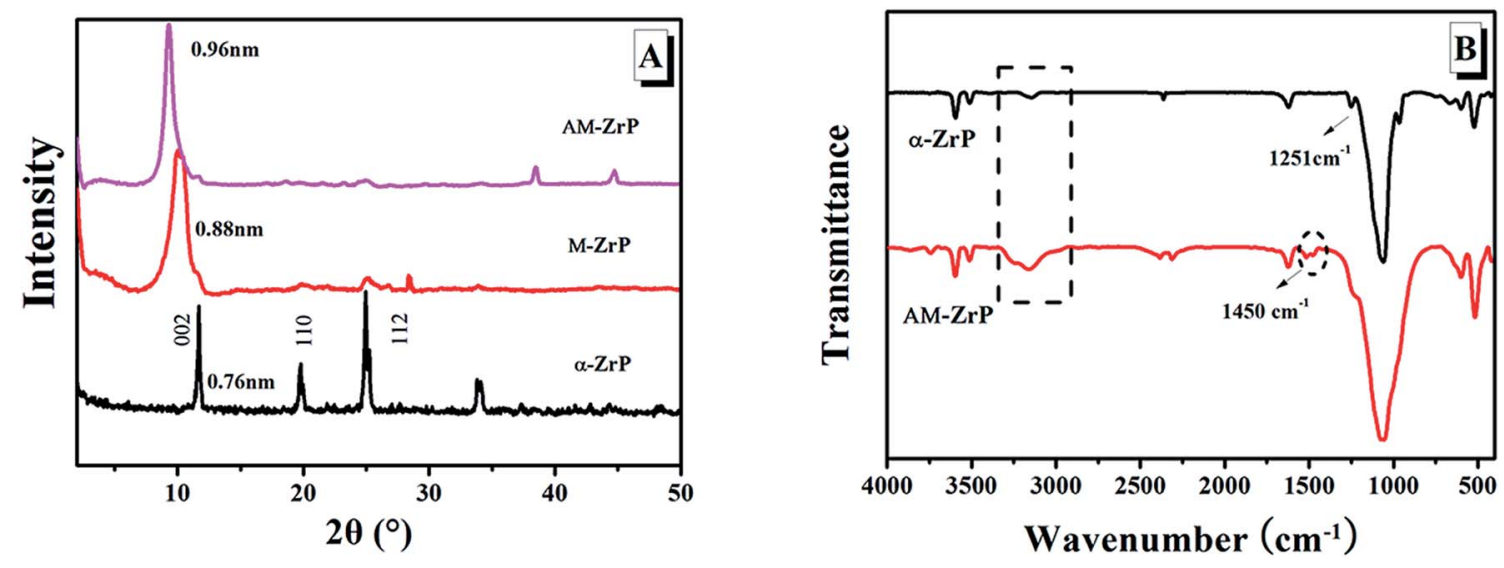

Fig. 4 (A) XRD and (B) FTIR spectra of $\alpha$-ZrP and AM-ZrP. 

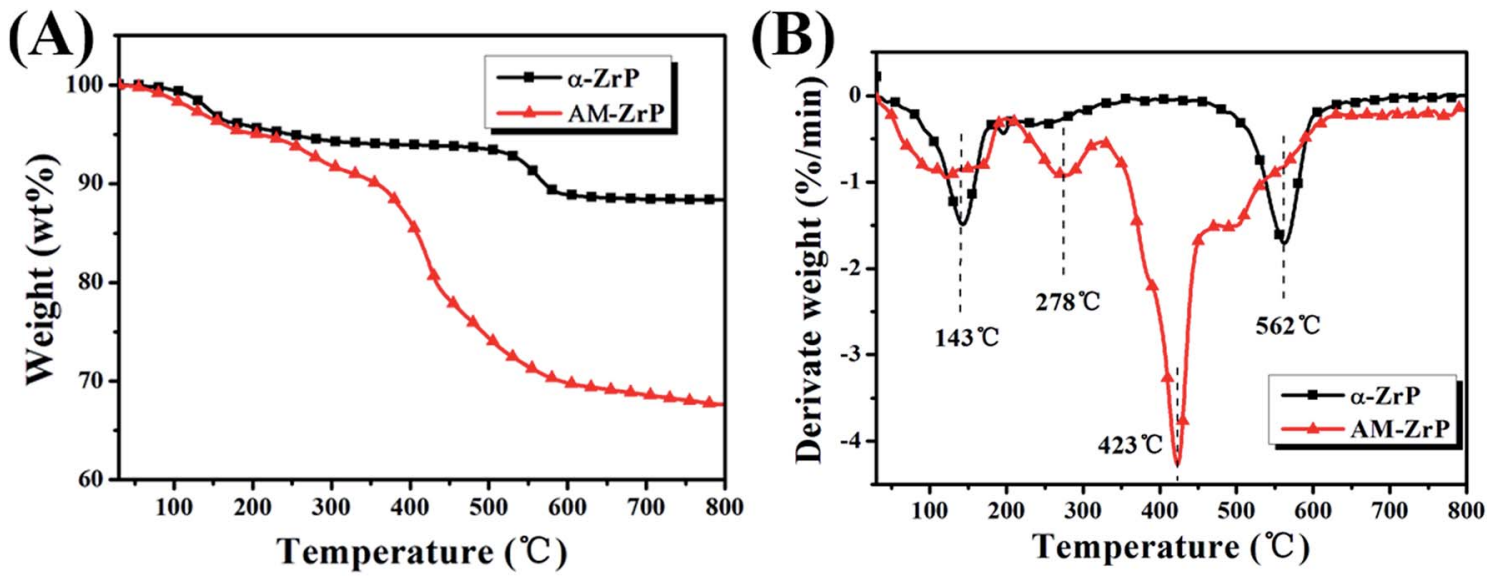

Fig. 5 (A) TGA and (B) differential thermogravimetric analysis (DTG) curves of $\alpha$-ZrP and AM-ZrP under air atmosphere.

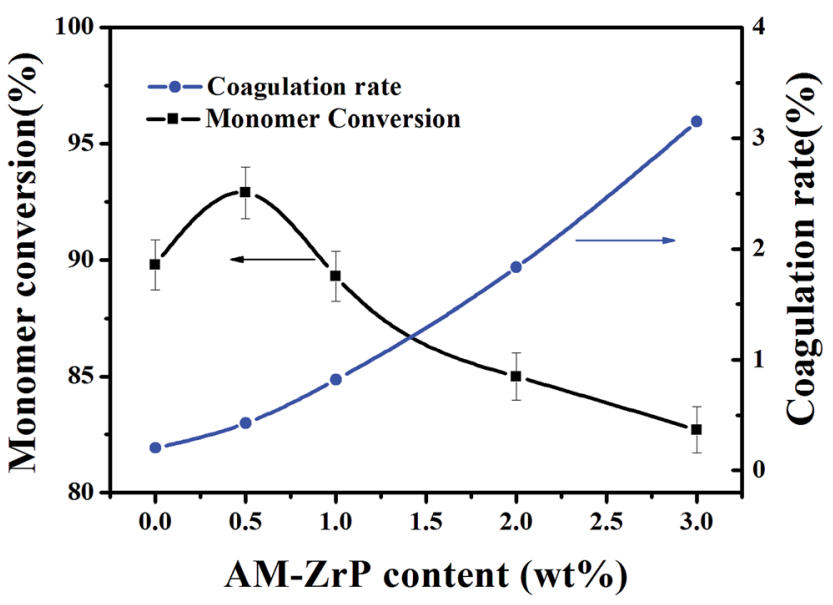

Fig. 6 Effects of AM-ZrP content on the monomer conversion and coagulation rate.

a slight increase to $0.43 \mathrm{wt} \%$. With the increase of AM-ZrP content from $0.5 \mathrm{wt} \%$ to $3.0 \mathrm{wt} \%$, the monomer conversion gradually decreased from $92.9 \mathrm{wt} \%$ to $82.7 \mathrm{wt} \%$, while the coagulation rate greatly enhanced from $0.43 \mathrm{wt} \%$ to $3.15 \mathrm{wt} \%$. As the AM intercalated into the AM-ZrP would be initiated during polymerization, at low AM-ZrP content, the introduction of AM-ZrP had a little effect on the monomer conversion. However, with the increase of AM-ZrP content, the AM-ZrP nanoplatelets got together as big aggregates and prevented the monomers from copolymerizing, resulting in the decrease of monomer conversion. Moreover, the AM-ZrP aggregates increased the probability of collisions between particles, leading to the high precipitate and coagulation rate during polymerization.

\subsection{Structure and morphology PPA/AM-ZrP}

Fig. 7 presents the XRD spectra of PPA and PPA/AM-ZrP composites. The spectrum of PPA/AM-ZrP-1 was similar to that of the PPA, and no diffraction peaks could be seen from the spectrum, indicating that the AM-ZrP nanoplatelets were well exfoliated in the PPA matrix. With the increase of AM-ZrP content from $0.5 \mathrm{wt} \%$ to $3.0 \mathrm{wt} \%$, a diffraction peak near $8.2^{\circ}$ gradually appeared in the spectra, and the diffraction peak became obvious and strong for the spectrum of PPA/AM-ZrP4, resulting from the poor dispersion of AM-ZrP nanoplatelets in PPA. Although the PPA/AM-ZrP composites were transparent shown in the inserted images, the transparency of PPA/AM-ZrP composites decreased with the increase of AM$\mathrm{ZrP}$ content. It could be derived that the AM-ZrP nanoplatelets were easy to aggregate at high content, which had a negative effect on the transparency of PPA/AM-ZrP composite.

The TEM images of $\alpha$-ZrP and PPA/AM-ZrP were shown in Fig. 8. As seen in Fig. 8(A and $\left.A_{1}\right)$, the dimension of the $\alpha$-ZrP nanoplatelets was about 120-160 nm with regular lamellar structures. For the PPA/AM-ZrP-1 in Fig. 8(B and $\left.\mathrm{B}_{1}\right)$, when the content of the AM-ZrP was $0.5 \mathrm{wt} \%$, the dimension of the AMZrP nanoplatelets decreased to approximately $80-120 \mathrm{~nm}$, indicating that the AM-ZrP nanoplatelets were exfoliated. Due to the in situ emulsion polymerization, the AM-ZrP nanoplatelets were covered by a layer of PPA polymer and well

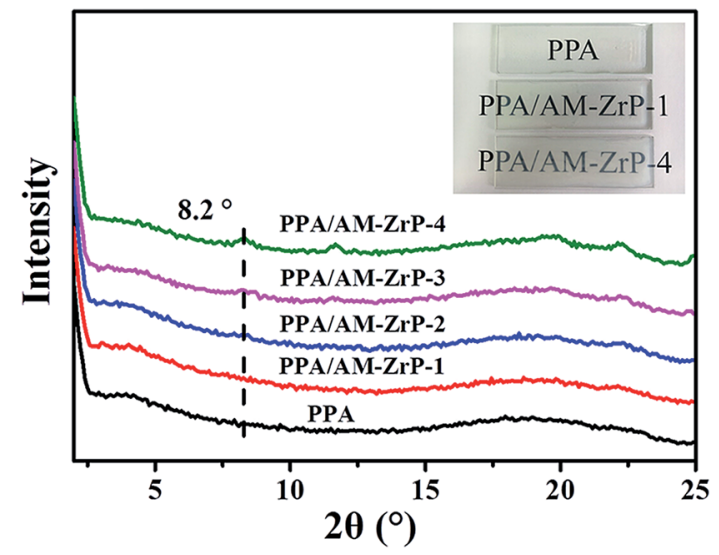

Fig. 7 XRD spectra of PPA and PPA/AM-ZrP. 

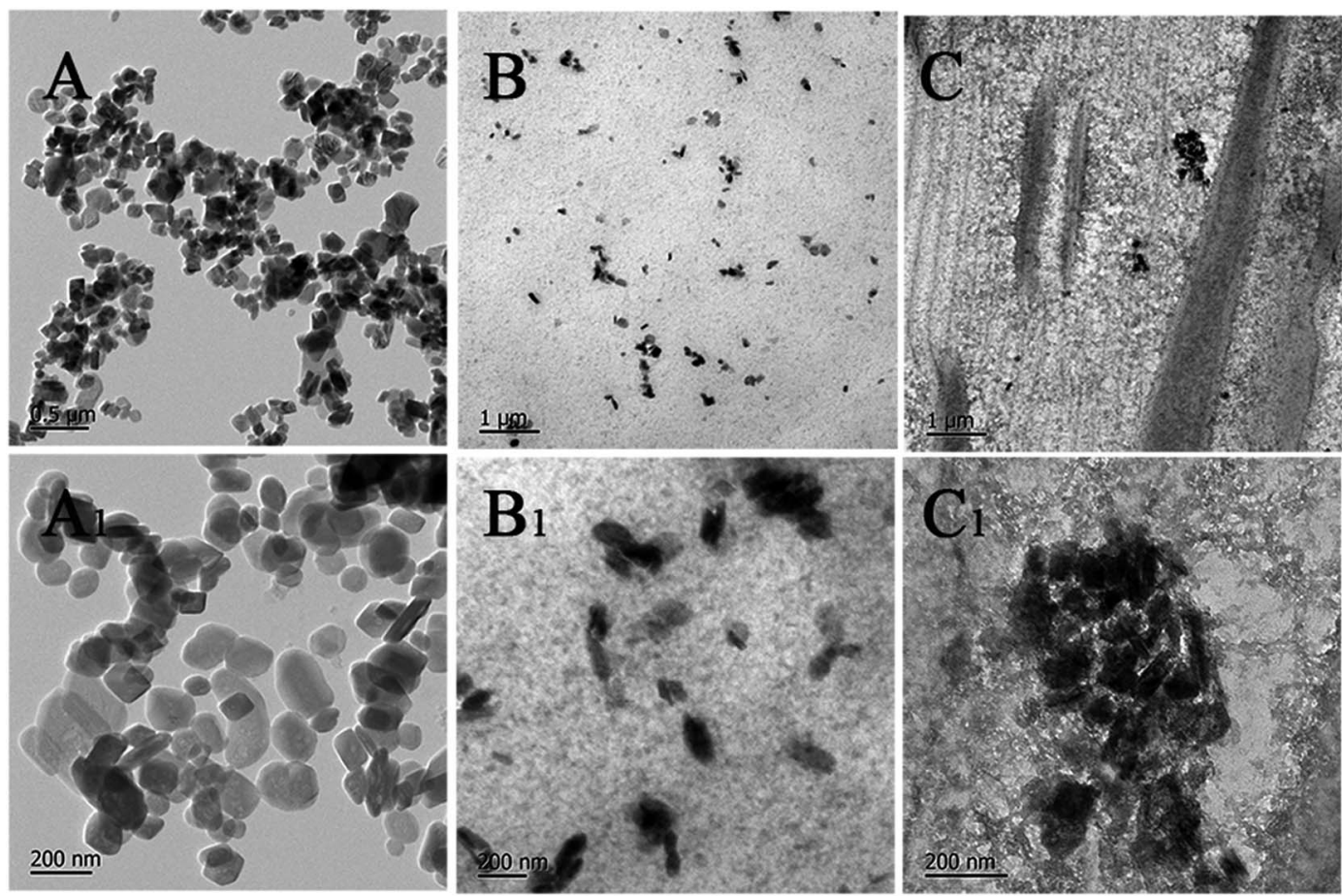

Fig. 8 TEM images of $\alpha$-ZrP and PPA/AM-ZrP. $\left(A\right.$ and $\left.A_{1}\right): \alpha-Z r P ;\left(B\right.$ and $\left.B_{1}\right)$ : PPA/AM-ZrP-1; $\left(C\right.$ and $\left.C_{1}\right)$ : PPA/AM-ZrP-4.

dispersed in the PPA matrix. With the increase of AM-ZrP content to $3.0 \mathrm{wt} \%$, in Fig. $8\left(\mathrm{C}\right.$ and $\left.\mathrm{C}_{1}\right)$, the AM-ZrP nanoplatelets got together as big aggregates because of the high specific surface areas and showed poor dispersion in the PPA, which might deteriorate the mechanical properties and flame retardancy of the composite.

\subsection{Thermal properties of PPA and PPA/AM-ZrP}

The DSC curves of the nanocomposite are presented in Fig. 9. As shown in Fig. 9, the PPA polymer chains possessed good flexibility with a $T_{\mathrm{g}}$ of about $8.9^{\circ} \mathrm{C}$. With the increase of AM$\mathrm{ZrP}$ content from $0.5 \mathrm{wt} \%$ to $3.0 \mathrm{wt} \%$, the $T_{\mathrm{g}}$ of PPA/AM-ZrP

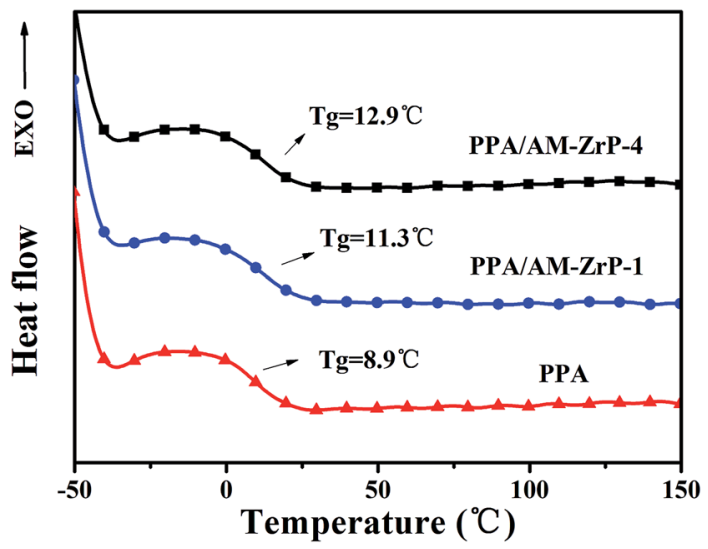

Fig. 9 DSC curves of PPA and PPA/AM-ZrP latexes film. increased from $11.3{ }^{\circ} \mathrm{C}$ to $12.9{ }^{\circ} \mathrm{C}$. It showed that the movement of the molecular chains was restricted by the exfoliated AM-ZrP nanoplatelets, leading to the high $T_{\mathrm{g}}$ of PPA/AM-ZrP.

Fig. 10 shows the TGA and DTG curves of PPA and PPA/AM$\mathrm{ZrP}$ under air atmosphere and the characteristic data are listed in Table 1. As shown in Fig. 9(A) and (B), the curves of TG and DTG of PPA and PPA/AM-ZrP were similar, indicating that the incorporation of AM-ZrP has a little effect on the thermal stability of composite. In Table 1, it could be seen that the $5 \mathrm{wt} \%$ weight loss temperature $\left(T_{5}\right.$ wt $\left.\%\right)$ of PPA was at about $295.6{ }^{\circ} \mathrm{C}$ and the max decomposition temperature $\left(T_{\max }\right)$ reached $417.9{ }^{\circ} \mathrm{C}$ with a maximum mass loss rate $\left(R_{\max }\right)$ of $33.64 \mathrm{wt} \% /$ min. When AM-ZrP was added into the PPA matrix, the $T_{\max }$ of the PPA/AM-ZrP composite increased to $419.6{ }^{\circ} \mathrm{C}$ and $420.5{ }^{\circ} \mathrm{C}$, and the $R_{\max }$ had a slight decrease of $1.54 \mathrm{wt} \% \min ^{-1}$ and $3.11 \mathrm{wt} \% \mathrm{~min}^{-1}$ for PPA/AM-ZrP-1 and PPA/AM-ZrP-4, respectively, which suggested that the incorporation of AM-ZrP was favorable for the increase of thermal stability of the composite to a certain extent. Meanwhile, as the AM-ZrP nanoplatelets possessed good thermal stability, with the increase of AM-ZrP content, the residue of the PPA/AM-ZrP composite at $700{ }^{\circ} \mathrm{C}$ increased.

\subsection{Effects of AM-ZrP on the flame retardancy}

Fig. 11 shows the heat release rate (HRR) curves and the corresponding parameters, and the LOI values of the PPA and PPA/ AM-ZrP. It could be seen that the peak heat release rate (pHRR) and total heat release (THR) of the PPA were $372 \mathrm{~kW} \mathrm{~m}^{-2}$ and $85 \mathrm{MJ} \mathrm{m}^{-2}$, respectively. When $0.5 \mathrm{wt} \%$ of AM-ZrP was added, 

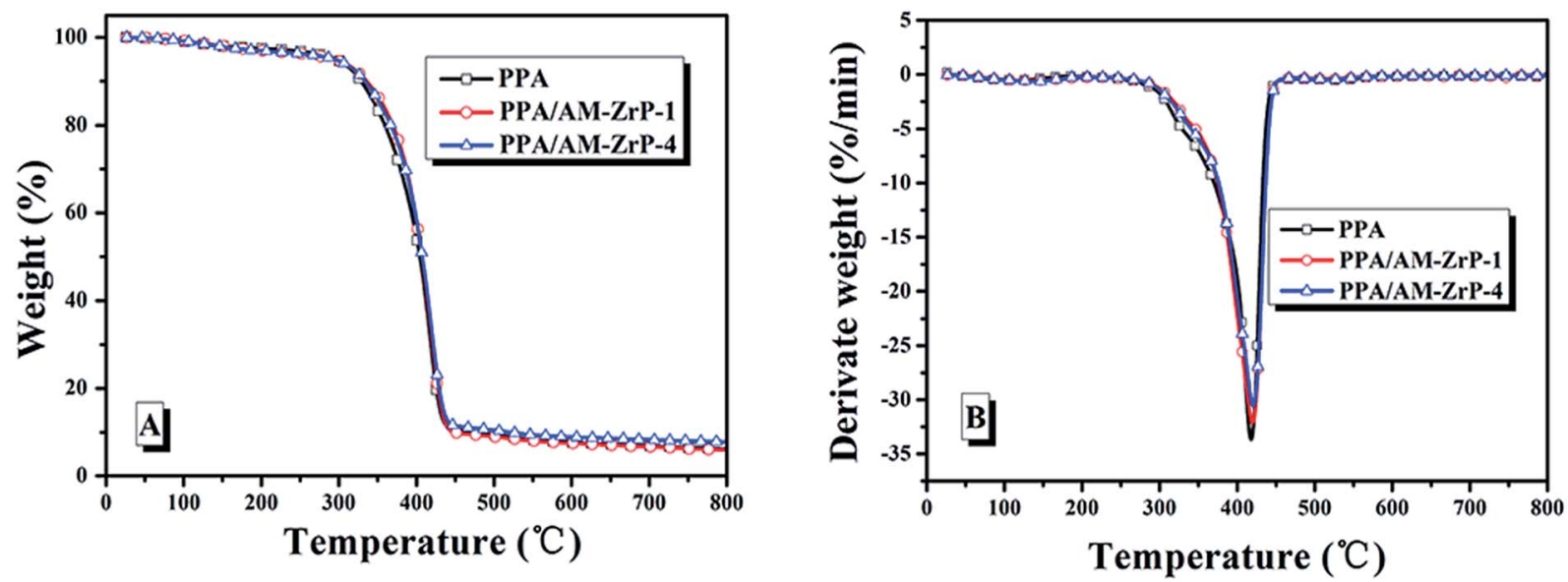

Fig. 10 (A) TGA and (B) DTG curves of PPA and PPA/AM-ZrP under air atmosphere.

Table 1 TGA and DTG data of PPA and PPA/AM-ZrP

\begin{tabular}{lllll}
\hline Sample & $T_{5} \mathrm{wt} \%\left({ }^{\circ} \mathrm{C}\right)$ & $T_{\max }\left({ }^{\circ} \mathrm{C}\right)$ & $\begin{array}{l}R_{\max } \\
\left(\mathrm{wt} \% \mathrm{~min}^{-1}\right)\end{array}$ & $\begin{array}{l}\text { Residue at } \\
700{ }^{\circ} \mathrm{C}(\mathrm{wt} \%)\end{array}$ \\
\hline PPA & 295.6 & 417.9 & 33.64 & 6.69 \\
PPA/AM-ZrP-1 & 292.7 & 419.6 & 32.10 & 6.87 \\
PPA/AM-ZrP-4 & 294.0 & 420.5 & 30.53 & 8.28
\end{tabular}

the pHRR and THR of the PPA/AM-ZrP-1 decreased to $338 \mathrm{~kW}$ $\mathrm{m}^{-2}$ and $81.5 \mathrm{MJ} \mathrm{m}^{-2}$, respectively. Meanwhile, the LOI value of the PPA was $23.5 \%$, and it increased to $25.0 \%$ for PPA/AM-ZrP-1, indicating that the flame retardancy of the composite was improved. However, with the increase of AM-ZrP content to $3.0 \mathrm{wt} \%$, the pHRR increased to $352 \mathrm{~kW} \mathrm{~m}^{-2}$ and the THR enhanced to $91.3 \mathrm{MJ} \mathrm{m}^{-2}$, even higher than that of the PPA. Moreover, the LOI value of the PPA/AM-ZrP-4 decreased to $24.0 \%$. The CCT results were consistent with LOI test, which could be explained as follows. On one hand, the layer structure of the AM-ZrP would restrict the activity of polymer chains, leading to the increase of thermal stability of the composite.

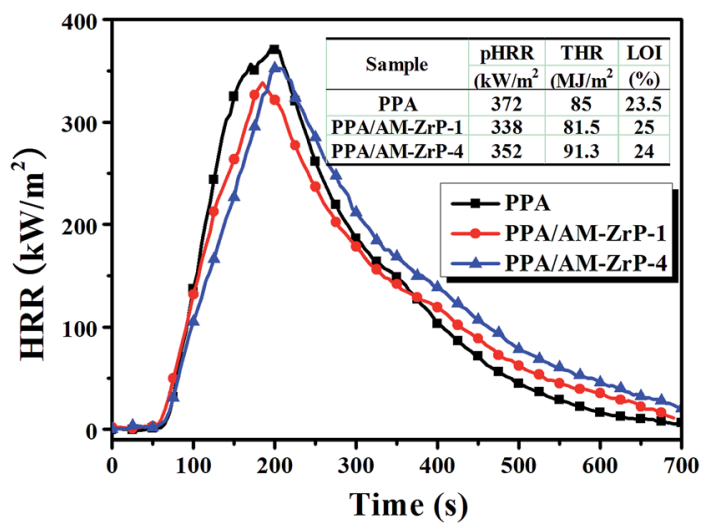

Fig. 11 Heat release rate (HRR) curves and LOI values of PPA and PPA/ AM-ZrP
Moreover, the AM-ZrP could promote the formation of dense carbon layer during combustion. On the other hand, as the AMZrP nanoplatelets possessed a dimension of 100-200 $\mathrm{nm}$, these special flake-like and nano-scale structures made the AM-ZrP acted as physical barriers to prevent heat and oxygen transmission during combustion. For the PPA/AM-ZrP with low AMZrP content, the AM-ZrP nanoplatelets were exfoliated and well dispersed in the PPA substrate, which was favour for the ordered arrangement of AM-ZrP. However, with the increase of AM-ZrP content, the AM-ZrP nanoplatelets were easy to get together as aggregates due to the high specific surface areas during polymerization and showed poor dispersion in the composite, resulting in the deteriorated flame retardancy. Therefore, the dispersion of the AM-ZrP nanoplatelets was extremely important for the flame retardant properties of the PPA/AM-ZrP composite.

The char layers of PPA and PPA/AM-ZrP-1 after CCT test were taken for the SEM and EDS test, and the results are presented in Fig. 12. In Fig. 12(A), the surface of the char layer of PPA was rather rough and fluffy with many irregular cracks and holes distributing on it, indicating that there was a large amount of gases escaped during combustion. While in Fig. 12(B), a dense and compact char layer was seen and a few holes could be observed from the surface of PPA/AM-ZrP-1, which could prevent the permeation of decomposed gases through the char layer. Moreover, the compact char layer acted as barriers to segregate the transportation of heat, oxygen and combustible gas, resulting in the reduction of the heat release, mass loss and smoke production. From Fig. $12\left(\mathrm{~A}_{1}\right)$, the char layer was mainly composed of $\mathrm{C}, \mathrm{O}$ and $\mathrm{P}$ elements and weight percent were about $25.13 \%, 46.43 \%$ and $18.76 \%$, respectively. While in Fig. $12\left(\mathrm{~B}_{1}\right)$, the element of $\mathrm{Zr}$ appeared on the surface of char layer of the PPA/AM-ZrP-1, and the weight percent of $\mathrm{C}$ element increased to $41.81 \%$, much higher than that of the PPA. The EDS analysis suggested that the AM-ZrP in the PPA/AM-ZrP composite acted as catalyst for the char layer production, and promoted the formation of compact char layer, resulting in the good flame retardancy of the composite..$^{33,34}$ 

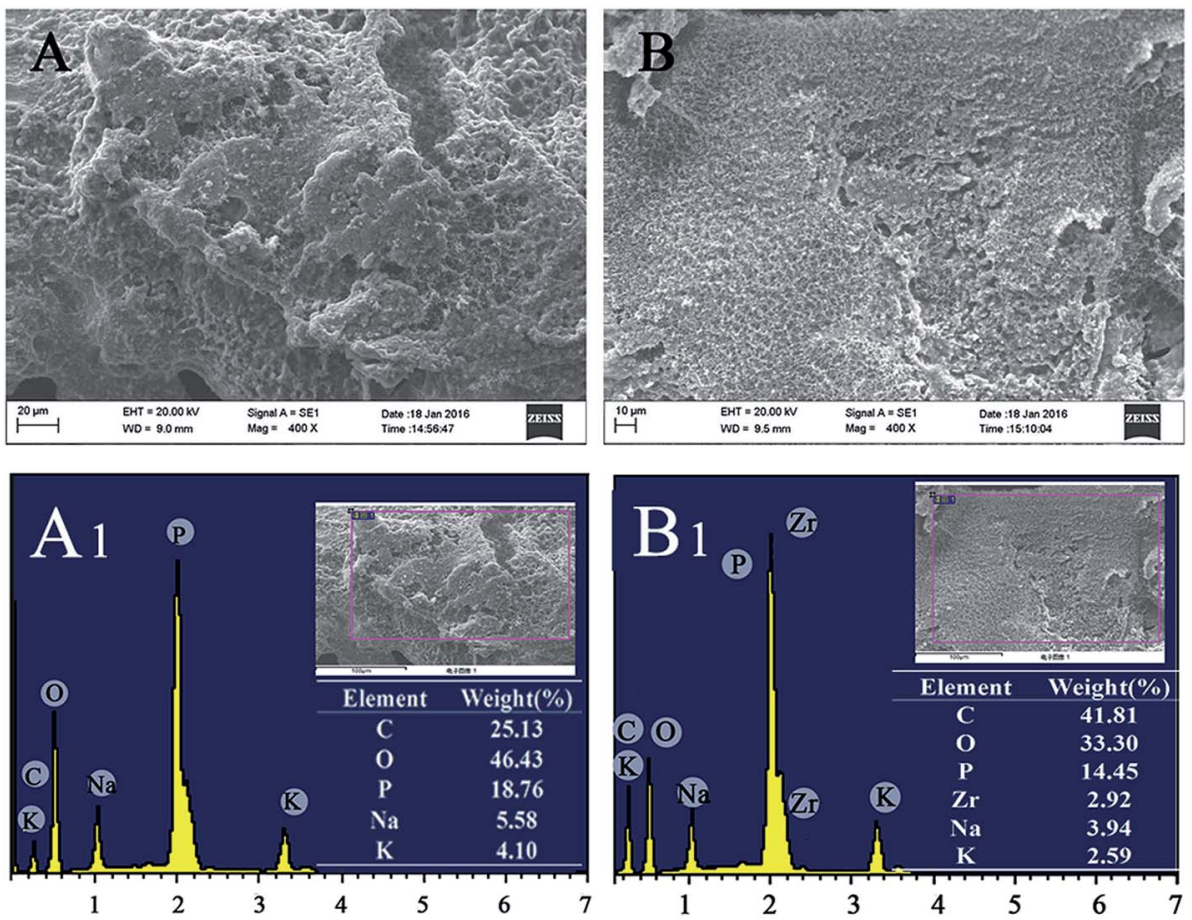

Fig. 12 SEM images and EDS spectra of the char layer for $\left(A\right.$ and $\left.A_{1}\right)$ PPA and (B and $\left.B_{1}\right)$ PPA/AM-ZrP-1.

\section{Conclusion}

The AM-ZrP was successfully prepared by the intercalation of acrylamide into ZrP nanoplatelets, and was utilized to synthesize PPA/AM-ZrP nanocomposite via in situ emulsion polymerization. Results showed that the incorporation of AM-ZrP had a negative effect on the polymerization stability of the composite emulsion, and would increase the $T_{\mathrm{g}}$ of the composite. When $0.5 \mathrm{wt} \%$ of AM-ZrP was added, the AM-ZrP nanoplatelets were exfoliated and covered by the PPA polymer, and well dispersed in the PPA matrix. Meanwhile, the PPA/AMZrP-1 had lower pHRR and higher LOI than the PPA, indicating that the flame retardancy of the PPA/AM-ZrP-1 composite was improved. With the increase of AM-ZrP content to $3.0 \mathrm{wt} \%$, the AM-ZrP nanoplatelets got as big aggregates and showed poor dispersion in the composite, which had adverse effects on the flame retardancy of the composite. SEM and EDS analysis suggested that the existence of AM-ZrP in the composite could catalyze carbonization process, and promoted the formation of compact char layer, which was beneficial for the improvement of the flame retardancy of the composite.

\section{Conflicts of interest}

There are no conflicts to declare.

\section{Acknowledgements}

Thanks for the financial supports from the National Natural Science Foundation of China (51773064), Guangzhou Science and Technology Plan Project (201604010056) and the Natural Science Foundation of Guangdong Province (2017A030313303).

\section{References}

1 V. D. Athawale and M. A. Kulkarni, Prog. Org. Coat., 2009, 65, 392-400.

2 Y. Bao, J. M. Ma, X. Zhang and C. H. Shi, J. Master. Sci., 2015, 50, 6839-6863.

3 P. X. Chen, X. R. Zeng, H. Q. Li, X. D. Liu, D. D. Liu and X. L. Li, J. Appl. Polym. Sci., 2012, 124, 4694-4701.

4 D. G. Gao, W. B. Zhang, J. Z. Ma, C. M. Li and L. J. Zhang, J. Coat. Technol. Res., 2015, 12, 997-1004.

5 L. Zhang, Y. J. Cao, L. Wang, L. Shao and Y. P. Bai, RSC Adv., 2014, 4, 47708-47713.

6 F. Yang, W. Yang, L. Q. Zhu, Y. C. Chen and Z. M. Ye, Prog. Org. Coat., 2016, 95, 1-7.

7 S. W. Zhu and W. F. Shi, Polym. Degrad. Stab., 2002, 75, 543547.

8 S. Y. Lu and I. Hamerton, Prog. Polym. Sci., 2002, 27, 16611712.

9 C. I. Lindsay, S. B. Hill, M. Hearn, G. Manton, N. Everall, A. Bunn, J. Heron and I. Fletcher, Polym. Int., 2000, 49, 1183-1192.

10 Q. Y. Liu, X. R. Zeng, H. Q. Li and X. J. Lai, Chem. Adhes., 2012, 34, 7, in Chinese.

11 F. T. Han, Q. Y. Liu, X. J. Lai, H. Q. Li and X. R. Zeng, Prog. Org. Coat., 2014, 77, 975-980.

12 C. M. Feng, Y. Zhang, S. W. Liu, Z. G. Chi and J. R. Xu, Polym. Degrad. Stab., 2012, 97, 707-714. 
13 P. A. Song, L. F. Tong and Z. P. Fang, J. Appl. Polym. Sci., 2008, 110, 616-623.

14 S. P. Kumar, S. Takamori, H. Araki and S. Kuroda, RSC Adv., 2015, 5, 34109-34116.

15 B. Sang, Z. W. Li, X. H. Li, L. G. Yu and Z. J. Zhang, J. Master. Sci., 2016, 51, 8271-8295.

16 S. Liu, H. Q. Yan, Z. P. Fang, Z. H. Guo and H. Wang, RSC Adv., 2014, 4, 18652-18659.

17 Y. Q. Lin, S. H. Jiang, Z. Gui, G. H. Li, X. X. Shi, G. H. Chen and X. F. Peng, RSC Adv., 2016, 6, 86632-86639.

18 C. X. Zhao, Z. Sun, B. L. Liu and G. Peng, J. Macromol. Sci., Part B: Phys., 2013, 52, 1453-1464.

19 R. Zhang, Y. Hu, J. Y. Xu, W. C. Fan and Z. Y. Chen, Polym. Degrad. Stab., 2004, 85, 583-588.

20 Z. D. Han, A. Fina and G. Malucelli, Prog. Org. Coat., 2015, 78, 504-510.

21 T. Jiang, C. H. Liu, L. Liu, J. Hong, M. Dong and X. Deng, RSC Adv., 2016, 6, 91720-91727.

22 L. Y. Sun, W. J. Boo, R. L. Browning, H. J. Sue and A. Clearfield, Chem. Mater., 2005, 17, 5606-5609.

23 L. F. Xu, C. H. Lei, R. J. Xu, X. Q. Zhang and F. Zhang, Polym. Degrad. Stab., 2016, 133, 378-388.
24 W. Y. Xing, P. Zhang, L. Song, X. Wang and Y. Hu, Mater. Res. Bull., 2014, 49, 1-6.

25 J. Alongi and A. Frache, Polym. Degrad. Stab., 2010, 95, 19281933.

26 X. Q. Liu, D. Y. Wang, X. L. Wang, L. Chen and Y. Z. Wang, Polym. Degrad. Stab., 2011, 96, 771-777.

27 L. L. S. Brandao, L. C. Mendes, M. E. Medeiros, L. Sirelli and M. L. Dias, J. Appl. Polym. Sci., 2006, 415, 3868-3876.

28 H. D. Lu and C. A. Wilkie, Polym. Adv. Technol., 2011, 22, 1123-1130.

29 L. Y. Sun, W. J. Boo, H. J. Sue and A. Clearfield, New J. Chem., 2007, 31, 39-43.

30 J. S. Xu, Y. Tang, H. Zhang and Z. Gao, J. Inclusion Phenom. Macrocyclic Chem., 1997, 27, 303-317.

31 R. Zhang, Y. Hu, B. G. Li, Z. Y. Chen and W. C. Fan, J. Mater. Sci., 2007, 42, 5641-5646.

32 M. E. S. R. Silva, E. R. Dutra, V. Mano and J. C. Machado, Polym. Degrad. Stab., 2000, 67, 491-495.

33 H. D. Lu, C. A. Wilkie, M. Ding and L. Song, Polym. Degrad. Stab., 2011, 96, 1219-1224.

34 L. F. Xu, C. H. Lei, R. J. Xu, X. Q. Zhang and F. Zhang, RSC Adv., 2016, 6, 77545-77552. 